\title{
Breaking through the attentional window: Capture by abrupt onsets versus color singletons
}

\author{
Nicholas Gaspelin • Eric Ruthruff • Mei-Ching Lien • Kyunghun Jung
}

Published online: 18 July 2012

(C) Psychonomic Society, Inc. 2012

\begin{abstract}
Theeuwes (Psychonomic Bulletin \& Review 11:65-70, 2004) proposed that stimulus-driven capture occurs primarily for salient stimuli that fall within the observer's attentional window, such as when performing a parallel search. This proposal, which is supported by some studies, can explain many seemingly discrepant results in the literature. The present study tested this proposal using a modified precuing paradigm. Search mode was manipulated via target-distractor similarity in color space. In the parallel search condition, the orange target "popped out" from a set of distantly colored distractors (blue and green). In the serial search condition, the orange target was more difficult to find amongst a set of similarly colored distractors (yellow and red). In Experiments 1 and 2, cue validity effects for irrelevant-color singleton cues were greater under parallel than under serial search, at least partially replicating previous studies favoring the attentional-window account (e.g., Belopolsky, Zwaan, Theeuwes, \& Kramer, Psychonomic Bulletin \& Review 14:934-938, 2007). We found the opposite pattern, however, for capture by abrupt onsets (Experiments 3 and 4), in which case capture effects were actually greater under serial search. In sum, parallel search appears to facilitate capture by color singletons, yet to inhibit capture by abrupt onsets.
\end{abstract}

Keywords Attentional capture - Visual search - Spatial attention

\footnotetext{
N. Gaspelin $(\bowtie) \cdot$ E. Ruthruff $\cdot$ K. Jung

Department of Psychology, University of New Mexico, MSC03 2220,

Albuquerque, NM 87131-1161, USA

e-mail: gaspelin@unm.edu

M.-C. Lien

Oregon State University,

Corvallis, OR, USA
}

Sometimes, task-irrelevant information draws our attention. While driving, for example, a bright billboard advertisement might draw attention, seemingly against our will. Yet at other times, very salient information fails to capture our attention: A waving pedestrian (or, classically, a waving gorilla) may go unnoticed (Simons \& Chabris, 1999). Indeed, one might wonder how a person distracted by every salient stimulus (e.g., flashing police beacons, brake lights, blinking crosswalk signs, or neon traffic cones) could possibly survive a single trip to the grocery store. These simple observations raise the question of how involuntary shifts of attention are guided. Can certain "super" stimuli capture our attention at any moment (bottom up)? Or are these shifts involuntary and yet, counterintuitively, driven by what we are looking for (top down)?

Research on attention capture has made great strides in identifying laboratory scenarios in which salient stimuli do and do not capture attention. However, opinions are still sharply divided about how to reconcile the puzzling empirical discrepancies from different paradigms and different types of salient stimuli. Theeuwes $(2004,2010)$ has proposed one promising reconciliation, in which stimulus-driven capture occurs only when objects are searched in parallel. This claim, if correct, would have important theoretical implications, as well as important practical implications for identifying realworld scenarios that leave an operator vulnerable to irrelevant capture. Although several findings have been suggestive (e.g., Belopolsky, Zwaan, Theeuwes, \& Kramer, 2007; Schreij, Owens, \& Theeuwes, 2008; Schreij, Theeuwes, \& Olivers, 2010), this claim has not yet been thoroughly tested. For this article, therefore, we used a precuing paradigm to assess whether differences between search modes (parallel vs. serial) can actually explain the discrepant findings in the attentionalcapture literature. Before describing the specifics of our approach, we will first review previous evidence for capture by salient objects and the role of search mode. 


\section{Stimulus-driven versus goal-driven capture of attention}

Stimulus-driven accounts of attentional capture have proposed that certain salient stimulus features guide attention, irrespective of the current goals. Feature singletons-stimuli with a unique feature appearing against a homogeneously featured background - for instance, are thought to be particularly salient, and are considered likely candidates for stimulus-driven capture. A lone green letter amongst several red letters, for example, would be a color singleton, as would a lone yellow daisy in a field of green grass. Abruptly appearing stimuli (called abrupt onsets) and moving stimuli are also thought to be particularly salient.

One of the most prominent variants of stimulus-driven capture is that proposed by Theeuwes $(1992,2004,2010)$, which is based on a zoom-lens model of spatial attention. To briefly summarize, zoom-lens theories assume that the spotlight of spatial attention (often called the attentional window) can change in size, focusing either narrowly or diffusely across a visual scene. Theeuwes proposed that relative salience within this attentional window guides subsequent focusing. In other words, any salient stimulus appearing inside the attentional window would subsequently capture attention, whereas those falling outside the window would not. The more diffuse the attentional window (as in parallel search), the more likely it is that a salient stimulus will fall within that window and thus capture attention. Because the size of the attentional window is under voluntary control, a participant could effectively avoid capture by shrinking the attentional window (as in serial search). Thus, search mode strongly determines whether salient stimuli will capture attention involuntarily.

Theeuwes (1992) provided initial support for this claim using a paradigm that explicitly encouraged a diffuse attentional window. In this additional-singleton paradigm, participants searched an array of items for a singleton target, such as a diamond target amongst circle distractors. Because the singleton target "popped out" of the display, it was assumed that participants would search the displays in parallel, with a diffuse attentional window. Meanwhile, a taskirrelevant color singleton distractor was sometimes presented. Although participants were instructed to ignore this color singleton distractor, they often produced longer response times (RTs) when it was present than when it was absent. This present-absent cost was taken as evidence that the distractor captured attention, temporarily drawing attention away from the target.

Recently, Belopolsky et al. (2007) provided even more direct support for the attentional-window account using a go/no-go paradigm (for a related study, see also Belopolsky $\&$ Theeuwes, 2010). In this paradigm, participants searched triangular arrays of letters for a target. In the diffuse window condition, participants first identified the orientation of the triangular array. If the triangular search array pointed upward, the participant searched the array of letters for the target (go trial). If the triangular search array pointed downward, the participant skipped to the next trial (no-go trial). Presumably, participants spread their attentional window across the entire search display in order to ascertain which way the large triangular array was pointing. In the focused window condition, participants used the shape of the small triangular fixation point, located at screen center, to determine whether the current trial was a go or no-go trial. Presumably, this encouraged a very narrow attentional window. In both of these search conditions, a nonpredictive color singleton appeared on every trial at either a target (valid) or distractor (invalid) location. The critical finding was that participants showed validity effects, indicating capture, only under the diffuse-window condition. This pattern was taken to support Theeuwes's attentionalwindow account of capture.

Unlike stimulus-driven accounts, goal-driven accounts of attentional capture claim that involuntary shifts depend on what the participant is looking for-the contingent involuntary-orienting hypothesis (e.g., Folk, Remington, \& Johnston, 1992). According to this theory, participants establish an attentional goal (often called an attentional set) for the feature distinguishing the target from the rest of the display. If a stimulus matches this attentional set, it will capture attention. In sum, top-down control settings determine attentional capture.

In a classic experiment, Folk et al. (1992) provided evidence for their theory using a precuing paradigm. Participants searched for either an abruptly onsetting target or a red target in different blocks of trials. This search display was preceded by a nonpredictive cue that was either an abrupt onset or red. The cue location could be invalid (different than the target), valid (same as the target), or neutral (the cue was absent). Also, it could either match or mismatch the distinguishing feature of the target (red or onset). If attention were captured by the cues, target responses should be faster for valid cues and slower for invalid cues (called cue validity effects). Critically, participants showed cue validity effects only for cues matching the target feature. For example, onset cues produced cue validity effects only when participants looked for onset targets, not red singleton targets. These results suggest that attentional capture, although rapid, stimulus-triggered, and apparently involuntary, is nevertheless entirely contingent on the viewer's top-down goals.

The debate between these two competing theories of attentional capture has not yet been resolved. As a reconciliation, stimulus-driven theorists have argued that precuing paradigms discourage capture by encouraging a focused, serial search (Theeuwes, 2004). This hypothesis is quite plausible: When participants search serially, attentional 
allocation might be primarily determined by proximity from the previous locus of attention (or a fixed search path) rather than by salience. Or perhaps slower searches allow more time for top-down task relevance to overcome bottom-up salience. Although highly promising, this attentionalwindow hypothesis has not yet been thoroughly tested, especially in the precuing paradigm, which is the purpose of the present study. Before describing our approach, it will be helpful to first review what exactly is meant by "serial" and "parallel" search.

\section{Parallel versus serial search}

Treisman and Gelade's (1980) feature-integration theory of attention prominently distinguished parallel and serial processes in visual search. These researchers noted that targets defined by a single feature seem to "pop out" of the search display (called feature search). For example, a lone blue letter would certainly stand out in a display of red Ts and green Xs. In such displays, participants are often able to rapidly report the presence or absence of a target, independent of display set size. Treisman and Gelade claimed that, in feature searches, all locations are searched in parallel.

Targets defined by a conjunction of features (i.e., conjunction search) are considerably more difficult to find. Referring to the previous example, a green $\mathrm{T}$ would not stand out in a display containing red Ts and green Xs. Here, the time required to report the presence or absence of a target increases sharply with increasing display set size (this RT by set size function is often called the search slope). Moreover, search slopes are often roughly twice as steep on target-absent as on target-present trials. This finding is exactly what one would expect from a serial, self-terminating search because, on average, only half of the items are searched when the target is present, but all are searched when the target is absent. Treisman and Gelade (1980) reasoned that, in conjunction searches, each potential target location is searched serially. They also claimed that search slopes could be used to distinguish parallel and serial search modes from one another. For parallel searches, increasing set size minimally increases RT (i.e., flat search slope). For serial searches, however, increasing set size strongly increases RT (i.e., steep search slope), usually in a roughly linear fashion.

Some researchers have criticized the strict parallel-serial search mode distinction, and instead have suggested emphasizing the degree of efficiency (described further in the Parallel Versus Serial Search Revisited section in the General Discussion). Although we are sympathetic to these positions, we assume here that the conditions commonly referred to as "parallel" and "serial" do in fact reflect very different ways of allocating attention, as is required by
Theeuwes's $(1991,2004,2010)$ reconciliation of the capture literature.

\section{The present study}

Theeuwes's hypothesis that capture occurs only under parallel search is plausible and consistent with several studies. At the same time, these supporting studies have had a few major shortcomings: Many of the studies have relied exclusively on modified versions of Theeuwes's additionalsingleton paradigm (e.g., Theeuwes, 1992, 1994). Thus, it is unclear whether results from this paradigm generalize to other paradigms, such as the precuing paradigm typically employed by goal-driven theorists. Of particular concern is that the present-absent costs typically calculated in Theeuwes's additional-singleton paradigm may reflect nonspatial filtering costs (Becker, 2007; Folk \& Remington, 1998) that is, a slower decision about where to move attentionrather than an actual shift of spatial attention. In contrast, cue validity effects in the precuing paradigm are a direct indication of actual shifts of spatial attention (Folk \& Remington, 2010).

Another major limitation of these previous studies is that they have exclusively examined capture by color singletons (e.g., Belopolsky \& Theeuwes, 2010; Belopolsky et al., 2007; Theeuwes, 1992). It is critical to establish whether the attentional-window account generalizes to all salient stimuli. Here, we distinguish between a strong and a weak version of the attentional-window account. The strong version of the attentional-window theory is a fundamental assumption about the nature of attentional capture, making no distinction between color singletons and onsets (e.g., Theeuwes, 1991, 1992, 2004). This seems to be what attentional-window theorists initially had mind. For example, Theeuwes (1991) stated that "in an unfocused state, attention covers the entire visual field, which suggests that abrupt onsets and offsets do attract attention similarly. When an endogenous cue enables one to 'zoom in' on a particular area, abrupt transients clearly outside the circumscribed area cease to attract attention" (p. 90). However, a weak version adds an amendment that parallel search is needed for color singletons, but not abrupt onsets. This weak version has been adopted in more recent studies (e.g., Belopolsky et al., 2007; Theeuwes, 2010).

To our knowledge, no researchers have explicitly assessed the effect of search mode on capture by abrupt onsets, so it is unclear whether the strong or weak version of attentional-window theory is correct. Many studies have indirectly suggested that abrupt onsets can capture attention even under serial search (Franconeri, Hollingworth, \& Simons, 2005; Franconeri \& Simons, 2003; Jonides \& Yantis, 1988; Lamy \& Egeth, 2003; Rauschenberger, 
2003; Schreij et al., 2008; Schreij et al., 2010), but they did not actually manipulate search mode or verify that a particular search mode was used. To resolve this issue, further experiments are needed.

In the present study, we tested whether parallel search enables capture by irrelevant salient stimuli in a precuing paradigm akin to that used by Folk et al. (1992). Using a single manipulation of search mode, we examined capture by color singletons (Exps. 1 and 2) and by abrupt onsets (Exps. 3 and 4). In Experiment 5, we removed the cue entirely, to verify that our manipulation of search mode was effective.

\section{Experiment 1}

In the present precuing task, participants searched an eight-item array for an orange target letter and reported its identity ( $\mathrm{T}$ or L); see Fig. 1. We manipulated search mode via distractor similarity with respect to the orange target letter. In our "parallel" search condition, the distractor colors (green and blue) were very far in color space from the orange target letter. In this condition, the target was highly salient and would "pop out" of the display. In our "serial" search condition, the distractor colors (red and yellow) were very close in color space to the target letter. Half of participants were assigned to the parallel condition, and the other half were assigned to the serial condition. Before the search array, a color singleton cued a potential target location. This color singleton precue could either match (relevant) or mismatch (irrelevant) the target color. When present, this cue was nonpredictive of target location (invalid on seven-eighths of trials and valid on one-eighth of trials).

If capture occurs only under parallel search, cue validity effects (defined as invalid RT minus valid RT) should occur for irrelevant color singleton cues only in the parallel search condition, but not the serial search condition. However, contingent-capture theory would predict negligible cue validity effects by irrelevant color singleton cues in either search condition.

\section{Methods}

Participants A group of 48 undergraduates from the University of New Mexico participated for course credit. Two of the participants in the serial search condition were excluded from the final data analysis because of unusually high error rates $(>20 \%)$. This meant that 22 participants were analyzed in the serial condition and 24 in the parallel condition. All participants in all experiments of this study had normal color vision, as assessed by the Ishihara color vision test, and selfreported normal or corrected-to-normal visual acuity.
Experiment 1

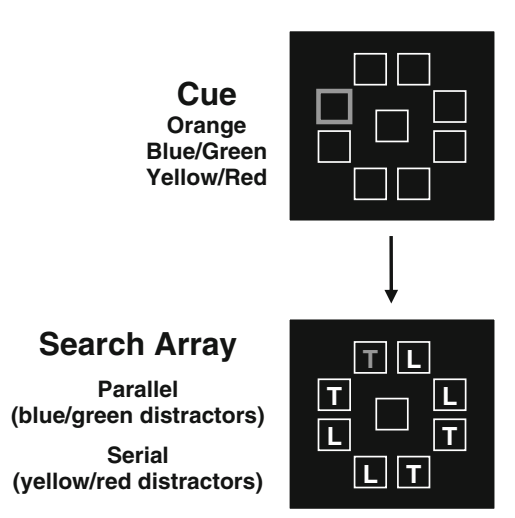

Experiment 2

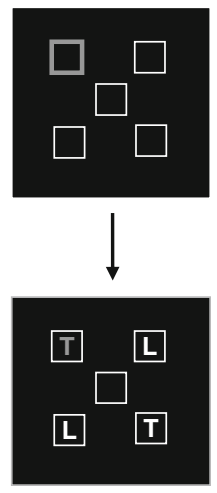

Fig. 1 Examples of the cues and search displays from Experiments 1 and 2. In Experiment 1, a set size of eight was used. In Experiment 2, a set size of four was used, which was similar to previous precuing experiments (cf. Folk et al., 1992). The displays, which contained colored items in the actual experiment, have been converted to a grayscale figure. In the actual precue displays, the gray box was colored (either orange, blue, green, yellow, or red). In the actual search arrays, the gray letter was orange, and the white letters were an even mixture of either green/blue (parallel) or yellow/red (serial). For the color figure, please visit our lab website: www.unm.edu/ ruthruff/PS color_figures.pdf

Apparatus A Dell personal computer displayed stimuli on 19-in. CRT monitors.

Stimuli E-Prime software (Psychology Software Tools Inc., Sharpsburg, PA) was used to design and present the stimuli, which were the letters $\mathrm{T}$ and $\mathrm{L}$ in Arial font. These letters were either green (RGB value of $0,153,0)$, red $(255,0,0)$, blue $(40,40,255)$, yellow $(255,205,0)$, orange $(255,130$, $0)$, or white $(255,255,255)$, designed to be of roughly equal luminance on a black background. The letters were $1.9^{\circ}$ in width and height, based on an average viewing distance of $60 \mathrm{~cm}$. The placeholders were white unfilled boxes $2.4^{\circ}$ in width and height. There were nine placeholders (eight around the potential target locations and one at fixation). These placeholders defined an imaginary rectangle $12.4^{\circ}$ in width and height. In the cue frame, one of the placeholder boxes served as a cue, which could be green, red, blue, yellow, or orange (same RGB values as those used for the letters in the target display); the remaining boxes were white.

Design Each search display contained four Ts and four Ls (see Fig. 1). The orange target letter's identity (T or L) was chosen at random. Display type (serial or parallel) was varied between participants. For parallel-search arrays, the distractors consisted of three green, three blue, and one 
white letter. For serial-search arrays, the distractors consisted of three yellow, three red, and one white letter. Color singleton precues were present on all trials and were nonpredictive of target location. The precue was valid on oneeighth of trials and invalid on seven-eighths of trials. The color of the cue was either the same as the target (i.e., orange; one-third of trials) or different (blue, green, yellow, or red; two-thirds of trials). Each participant first performed 72 practice trials divided into two blocks, and then 576 trials divided into eight blocks.

Procedure The participants were instructed to search for an orange $\mathrm{L}$ or $\mathrm{T}$ and to respond as quickly and accurately as possible by pressing the key labeled "L" or " $T$ " (actual keys: "c" or "b"). Participants were also instructed that the precue was nonpredictive of the target location and should be ignored. Each trial began with presentation of the nine placeholders for $1,000 \mathrm{~ms}$. This was followed by a blink of the central fixation placeholder for $100 \mathrm{~ms}$. Then the color singleton precue display appeared for $100 \mathrm{~ms}$, followed by another presentation of the placeholders for $50 \mathrm{~ms}$. The search array then appeared for $500 \mathrm{~ms}$ or until the participant made a response. The participants were given immediate accuracy feedback for $100 \mathrm{~ms}$ (a high tone for incorrect responses or no sound for correct responses). Participants also received block-by-block feedback on their mean RT and accuracy.

\section{Results and discussion}

Trials with RTs greater than $2,000 \mathrm{~ms}$ or less than $200 \mathrm{~ms}$ ( $0.7 \%$ of trials) were excluded from the RT and error rate analyses. Trials with an incorrect response were also excluded from the RT analyses. The resulting mean RTs and error rates are shown in Table 1 . Cue validity effects by condition are shown in Fig. 2.

A three-way analysis of variance (ANOVA) was conducted on mean RTs with the factors Search Condition (parallel vs. serial; between subjects), Cue Validity (invalid vs. valid; within subjects), and Cue Color (relevant vs. irrelevant; within subjects). This analysis revealed faster responses in the parallel condition $(521 \mathrm{~ms})$ than in the serial condition $(663 \mathrm{~ms}), F(1,44)=44.632, p<.001$, $\eta^{2}=.504$. This large effect suggests that our manipulation of search mode was effective.

Participants generally responded more slowly to the target following invalid cues $(614 \mathrm{~ms})$ than following valid cues $(570 \mathrm{~ms}), F(1,44)=100.942, p<.001, \eta^{2}=.696$. These overall cue validity effects did not differ between search conditions, $F(1,44)=0.090, p>.10, \eta^{2}=.002$. Participants responded slightly faster on trials in which the color singleton cue was relevant $(586 \mathrm{~ms})$ than when it was irrelevant (598 ms), $F(1,44)=25.845, p<.001, \eta^{2}=.370$. Participants
Table 1 Mean response times (RTs, in milliseconds) and percentage of errors (PEs) as a function of cue color (relevant vs. irrelevant color), search mode (parallel vs. serial), and cue validity (valid vs. invalid) for Experiment 1

\begin{tabular}{|c|c|c|c|c|c|c|}
\hline \multirow[t]{2}{*}{ Trial Type } & \multicolumn{2}{|c|}{ Valid } & \multicolumn{2}{|c|}{ Invalid } & \multicolumn{2}{|c|}{ Validity Effect } \\
\hline & $\mathrm{RT}$ & $\mathrm{PE}$ & RT & $\mathrm{PE}$ & RT & $\mathrm{PE}$ \\
\hline \multicolumn{7}{|l|}{ Serial } \\
\hline Relevant & 615 & $7.6 \%$ & 692 & $11.6 \%$ & $77^{*}$ & $4.0 \%$ \\
\hline Irrelevant & 667 & $11.3 \%$ & 676 & $10.9 \%$ & 9 & $-0.4 \%$ \\
\hline \multicolumn{7}{|l|}{ Parallel } \\
\hline Relevant & 486 & $5.5 \%$ & 549 & $8.8 \%$ & $63^{*}$ & $3.3 \%$ \\
\hline Irrelevant & 511 & $6.0 \%$ & 538 & $8.4 \%$ & $27^{*}$ & $2.4 \%$ \\
\hline
\end{tabular}

Validity effects were calculated as invalid minus valid. Asterisks indicate cue validity effects significantly greater than zero $(p<.05)$.

were slowed more by singleton cues under serial search $(18 \mathrm{~ms})$ than under parallel search $(7 \mathrm{~ms}), F(1,44)=5.241$, $p<.05, \eta^{2}=.106$. Cue validity effects were greater for relevant color singletons $(70 \mathrm{~ms})$ than for irrelevant color singletons (18 ms), $F(1,44)=95.282, p<.001, \eta^{2}=.694$.

The three-way interaction of search condition by cue validity by cue color was significant, $F(1,44)=9.256, p<.01$, $\eta^{2}=.174$. We followed up this interaction with an investigation of simple main effects. Preplanned $t$ tests revealed that cue validity effects from relevant color singletons did not differ significantly between search conditions $(63 \mathrm{~ms}$ for parallel and $77 \mathrm{~ms}$ for serial), suggesting that goal-driven capture was not affected by search mode, $t(44)=1.17, p>.10$.

The key question in this experiment was whether capture by irrelevant color singletons cues would be greater in the parallel search conditions. Cue validity effects for irrelevant color singletons were in fact significantly greater under parallel search $(27 \mathrm{~ms})$ than under serial search $(9 \mathrm{~ms}), t(44)=$ $2.18, p<.05$. Preplanned follow-up tests revealed that cue

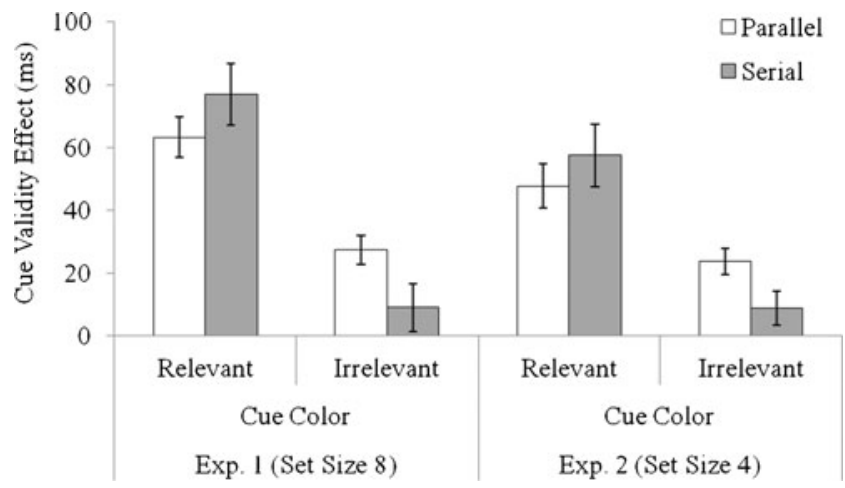

Fig. 2 Cue validity effects for relevant and irrelevant color singletons by search condition in Experiments 1 and 2. Cue validity effects were calculated as the invalid minus the valid mean. Bars represent the standard errors of the means 
validity effects were significant only under parallel search, $t(23)=6.06, p<.001$, not serial search, $t(21)=1.12, p>.10$ (see Table 1).

The same three-way mixed design ANOVA was conducted on mean error rates. Participants made significantly more errors following invalid cues $(9.9 \%)$ than following valid cues $(7.6 \%), F(1,44)=18.378, p<.001, \eta^{2}=.295$. These cue validity effects on error rates were greater for relevant cues $(3.7 \%)$ than for irrelevant color cues $(1.0 \%)$, $F(1,44)=7.204, p=.01, \eta^{2}=.141$. All other interactions and main effects were not significant.

To summarize, this experiment tested whether capture was greater under parallel than under serial search. Participants generally responded much more slowly in the serial than in the parallel search condition, suggesting that our manipulation of search mode was effective. Relevant color singleton cues (i.e., orange) produced large cue validity effects that did not vary much between search modes. However, irrelevant color singletons produced larger cue validity effects under parallel search than under serial search, replicating previous findings with color singletons (Belopolsky et al., 2007).

\section{Experiment 2}

In Experiment 1, we found that capture by irrelevant color singletons was greater under parallel search with a set size of eight. However, precuing paradigms typically use smaller set sizes (e.g., set size of four in Folk et al., 1992). In an attempt to more closely replicate such experiments, we reduced the set size to four in this experiment.

\section{Methods}

Participants A group of 58 new participants, drawn from the same participant pool as in Experiment 1, were in this experiment. Five of the participants were excluded from the final analysis because of unusually high error rates (more than $20 \%$ ). As a result, 25 participants in the serial condition and 28 in the parallel condition were included in the final analysis.

Apparatus, stimuli, and procedure The methods and stimuli were the same as in Experiment 1, except that the set size was reduced from eight to four. There were now five placeholders (four around the potential target locations and one at the fixation location). These placeholders were arranged in a square formation that was $10^{\circ}$ in width and height. The cue was presented on only half of the trials, because cue rarity is believed to encourage attentional capture (Neo \& Chua, 2006). When present, the cue was again nonpredictive of search location (25\% valid and $75 \%$ invalid).
Results and discussion

The data analysis was similar to that of Experiment 1. Application of the RT cutoffs (less than 200 or greater than $2,000 \mathrm{~ms}$ ) eliminated $0.3 \%$ of trials. The resulting mean RTs and error rates are shown in Table 2 . The cue validity effects by conditions are shown in Fig. 2.

First, to assess the search slopes of our search conditions, we compared the data from Experiment 1 (set size 8) and Experiment 2 (set size 4), collapsed across cue validity conditions. These data were analyzed with a two-way ANOVA with the between-subject factors Set Size (4 vs. 8) and Search Condition (parallel vs. serial). Participants responded more quickly in the parallel condition $(540 \mathrm{~ms})$ than in the serial condition $(624 \mathrm{~ms}), F(1,95)=37.388, p<$ $.001, \eta^{2}=.282$. Participants also responded more quickly at set size $4(556 \mathrm{~ms})$ than at set size $8(606 \mathrm{~ms}), F(1,95)=$ 13.292, $p<.001, \eta^{2}=.123$. Critically, the interaction between set size and search condition was significant, with participants producing steeper search slopes in the serial condition $(26.4 \mathrm{~ms})$ than in the parallel condition $(-1.1 \mathrm{~ms})$, $F(1,95)=15.679, p<.001, \eta^{2}=.143$. This classic interaction suggests that our manipulation of search mode was effective (see also Exp. 5).

A three-way ANOVA was conducted on the mean RTs from Experiment 2 with the factors Search Condition (parallel vs. serial; between subjects), Cue Validity (invalid vs. valid; within subjects), and Cue Color (relevant vs. irrelevant; within subjects). There was a trend for participants to respond more quickly in the parallel condition $(537 \mathrm{~ms})$ than in the serial condition $(567 \mathrm{~ms})$, although this difference did not reach significance, $F(1,51)=2.567, p>.10, \eta^{2}=.048$. This lack of significance reflects smaller effects of search condition at smaller set sizes, and hence less power (note that Exp. 1 had produced highly significant effects at larger set sizes; see also Exp. 3 below). Moreover, various interactions with search mode were significant, indicating that the search manipulation did, in fact, influence spatial attention.

Participants responded slightly more slowly on trials in which the singleton cue was an irrelevant $(554 \mathrm{~ms})$ rather than a relevant $(549 \mathrm{~ms})$ color, $F(1,51)=3.943, p<.06, \eta^{2}=.072$. The interaction of search condition and cue color was also significant, $F(1,51)=4.064, p<.05, \eta^{2}=.074$. A followup analysis revealed slower responses with irrelevant cues (572 $\mathrm{ms})$ than with relevant cues $(561 \mathrm{~ms})$ under serial search, $t(24)=3.08, p<.01$. However, RTs were similar with irrelevant cues $(537 \mathrm{~ms})$ and relevant cues $(537 \mathrm{~ms})$ under parallel search, $t(27)=0.02, p>.10$.

Participants responded more slowly on invalid trials $(569 \mathrm{~ms})$ than on valid trials $(535 \mathrm{~ms}), F(1,51)=74.813$, $p<.001, \eta^{2}=.595$. As in Experiment 1 , cue validity effects were greater for relevant color singletons $(53 \mathrm{~ms})$ than for 
Table 2 Mean response times (RTs, in milliseconds) and percentage of errors (PEs) as a function of cue color (target vs. nontarget color), search mode (parallel vs. serial), and cue validity (valid vs. invalid vs. absent) for Experiment 2

\begin{tabular}{|c|c|c|c|c|c|c|c|c|}
\hline \multirow[t]{2}{*}{ Trial Type } & \multicolumn{2}{|c|}{ Valid } & \multicolumn{2}{|c|}{ Invalid } & \multicolumn{2}{|c|}{ Absent } & \multicolumn{2}{|c|}{ Validity Effect } \\
\hline & RT & $\mathrm{PE}$ & RT & $\mathrm{PE}$ & RT & PE & RT & $\mathrm{PE}$ \\
\hline Serial & & & & & 568 & $9.3 \%$ & & \\
\hline Relevant & 533 & $6.6 \%$ & 590 & $8.9 \%$ & & & $57^{*}$ & $2.2 \%$ \\
\hline Irrelevant & 567 & $8.8 \%$ & 576 & $9.5 \%$ & & & 9 & $0.8 \%$ \\
\hline Parallel & & & & & 538 & $7.2 \%$ & & \\
\hline Relevant & 513 & $6.0 \%$ & 561 & $8.9 \%$ & & & $48^{*}$ & $2.9 \%$ \\
\hline Irrelevant & 525 & $7.4 \%$ & 549 & $7.5 \%$ & & & $24^{*}$ & $0.1 \%$ \\
\hline
\end{tabular}

Validity effects were calculated as invalid minus valid. Asterisks indicate cue validity effects significantly greater than zero $(p<.05)$.

irrelevant color singletons (16 ms), $F(1,51)=43.497, p<$ $.001, \eta^{2}=.46$. Overall, participants did not show significantly greater cue validity effects under parallel search (36 ms) than under serial search $(33 \mathrm{~ms}), F(1,51)=0.100$, $p>.10, \eta^{2}=.002$. Note that this effect is of little interest, because it is pooled across relevant and irrelevant cues, whereas we are primarily interested in the effects of irrelevant cues alone (see below).

The three-way interaction of search condition by cue validity by cue color was significant, $F(1,51)=5.009, p<$ $.05, \eta^{2}=.089$. This indicated that validity effects by irrelevant cues were dependent on search mode, while validity effects by relevant cues did not depend on search mode. We followed up this interaction with an investigation of simple main effects. Preplanned $t$ tests revealed that cue validity effects for relevant colors were not greater under parallel search (48 ms) than serial search (57 ms), $t(51)=0.819, p>$ .10. This finding suggests that relevant color singleton cues capture attention strongly, regardless of search mode.

The main question in this experiment was whether capture by irrelevant color singletons would be greater under parallel search, even at the smaller set sizes typically used in precuing paradigms. Indeed, the cue validity effects for irrelevant color singletons were again greater under parallel search $(24 \mathrm{~ms})$ than under serial search $(9 \mathrm{~ms}), t(51)=$ $2.238, p<.05$. Cue validity effects were significant under parallel search, $t(27)=5.86, p<.001$, but not under serial search, $t(24)=1.646, p>.10$. These data replicated the results in Experiment 1 with set size 8, and they suggest that irrelevant color singletons can capture attention only under parallel search in the precuing paradigm.

A three-way mixed design ANOVA was also conducted on mean error rates, with the factors Search Condition (parallel vs. serial; between subjects), Cue Validity (invalid vs. valid; within subjects), and Cue Color (relevant vs. irrelevant; within subjects). Participants made more errors on invalid trials $(8.6 \%)$ than on valid trials $(7.2 \%), F(1,51)=$ $5.270, p<.05, \eta^{2}=.094$. These cue validity effects on error rates were greater for relevant color singletons $(2.6 \%)$ than for irrelevant color singletons $(0.5 \%), F(1,51)=5.185 p<.05$, $\eta^{2}=.092$. All other main effects and interactions were nonsignificant.

To summarize, we replicated the main finding of Experiment 1-that task-irrelevant color singletons captured attention only under parallel search-using a smaller set size of four items. Both Experiments 1 and 2 generally support the claim that capture by taskirrelevant color singletons is possible only under parallel search (Belopolsky \& Theeuwes, 2010; Belopolsky et al., 2007; Theeuwes, 2004).

\section{Experiment 3}

In Experiments 1 and 2, we found capture by irrelevant color singletons only in the parallel search condition. However, it is unclear whether these results generalize to other types of salient stimuli. In Experiment 3, we examined capture by perhaps the most widely studied type of salient stimulus - abrupt onsets.

\section{Methods}

Participants A group of 61 new participants from the University of New Mexico participated in this experiment. Three of the participants were excluded from the analysis because of unusually high error rates (more than $20 \%$ ).

Apparatus, stimuli, and procedure The methods were mostly the same as in Experiments 1 and 2, except that white abrupt-onset cues were used instead of color cues (a change in the color of a box). The onset cues consisted of four white circles $\left(0.5^{\circ}\right.$ in diameter) surrounding one of the rectangular placeholders in the cue display (forming an imaginary diamond that was $3.3^{\circ}$ in height and width). The rectangular placeholders had been white in Experiments 1 and 2 but 
were changed to gray (RGB value: $138,138,138$ ) in this experiment to make the white onset dots more distinct. In order to discourage attentional set for onsets, we used premasks to make the search array consist entirely of offsets. The premasks were white rectangles with a central vertical line (whose segments could be deleted to reveal a $\mathrm{T}$ or $\mathrm{L}$ ). These masks appeared during the fixation and cue displays. Similarly, the blink previously denoting the beginning of a trial was also removed to remove any incentive to establish an attentional set for abrupt onsets.

Search array set size (4 or 8 ) was varied trial by trial. Every display had nine placeholders (two on each side and one in the center; see Fig. 3). In the set size 4 conditions, search array letters were spaced evenly across the entire display of eight locations, with each letter having an empty placeholder between it and another letter. The location of these four letters varied randomly across trials.

To increase statistical power, search display type (parallel vs. serial) was varied within participants. To reduce carryover effects, the experiment was divided into two session halves, one for each search condition; condition order was counterbalanced across participants. During each session half, the participants performed a practice block of 36 trials, followed by five blocks of 72 trials ( 360 total).

\section{Results and discussion}

The data analysis was similar to that of Experiments 1 and 2. Application of the RT cutoffs $(<200$ or $>2,000 \mathrm{~ms})$ eliminated $0.6 \%$ of the trials from the RT and error rate analyses.
Trials with incorrect responses were also excluded from the RT analyses. The resulting mean RTs and error rates are shown in Table 3, and the cue validity effects by condition are shown in Fig. 4.

First, we tested whether our manipulation of search mode was successful. For cue-absent trials, we conducted a two-way ANOVA on mean RTs with two factors: Search Condition (parallel vs. serial) and Set Size (4 vs. 8). Participants generally responded faster in the parallel condition $(569 \mathrm{~ms})$ than in the serial condition (671 ms), $F(1,57)=168.443, p<.001, \eta^{2}$ $=.747$. They also responded faster at set size $4(592 \mathrm{~ms})$ than at set size $8(648 \mathrm{~ms}), F(1,57)=295.014, p<.001, \eta^{2}=.838$. Most importantly, the participants produced steeper search slopes in the serial condition ( $20.8 \mathrm{~ms}$ per item) than in the parallel condition (7.5 ms per item), $F(1,57)=69.175, \quad p<$ $.001, \eta^{2}=.548$. This classic set size by search condition interaction on cue-absent trials suggests that our manipulation of search mode was in fact successful.

Second, for cue-present trials, we conducted a three-way within-subjects ANOVA on mean RTs with the factors Search Condition (parallel vs. serial), Cue Validity (invalid vs. valid), and Set Size (4 vs. 8). Again, participants performed faster in the parallel condition $(559 \mathrm{~ms})$ than the serial condition (662 ms), $F(1,57)=151.092, p<.001, \eta^{2}=.726$. They also responded faster at set size $4(586 \mathrm{~ms})$ than at set size $8(635 \mathrm{~ms}), F(1,57)=163.757, p<.001, \eta^{2}=.742$. Moreover, they showed steeper search slopes for the serial condition (18.4 ms per item) than for the parallel condition $(5.9 \mathrm{~ms}$ per item), $F(1,57)=63.187, p<.001, \eta^{2}=.526$. This also suggests that our manipulation of search strategy was successful.

\section{Experiment 3}

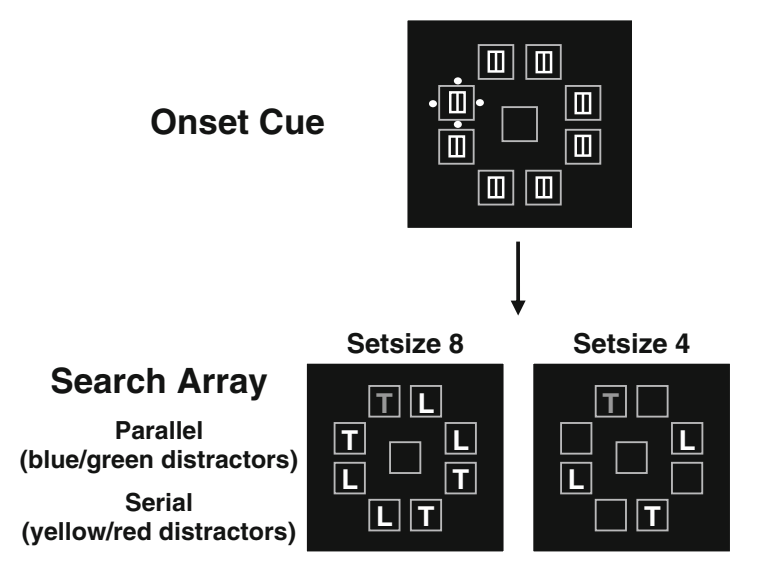

\section{Experiment 4}

\section{No Pre-Cue}

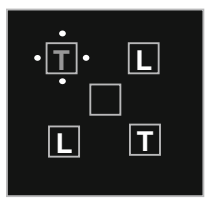

Fig. 3 Examples of the cues and search displays from Experiments 1 and 2. In Experiment 3, two different set sizes (four and eight) were used, and the cue appeared before the search array. In Experiment 4, the cue appeared simultaneously with the search display ( 0 -ms SOA), which is similar to many irrelevant-feature paradigms with abrupt onsets (cf. Jonides \& Yantis, 1988). The displays, which contained colored items in the actual experiment, have been converted to a grayscale figure. In the actual search arrays, the gray letter was orange, and the white letters were an even mixture of either green/blue (parallel) or yellow/red (serial). For the color figure, please visit www.unm.edu/ ruthruff/PS_color figures.pdf 
Table 3 Mean response times (RTs, in milliseconds) and percentages of errors (PEs) as a function of set size (4 vs. 8), search mode (parallel vs. serial), and cue validity (valid vs. invalid vs. absent) for Experiment 3

\begin{tabular}{|c|c|c|c|c|c|c|c|c|}
\hline \multirow[t]{2}{*}{ Trial Type } & \multicolumn{2}{|c|}{ Valid } & \multicolumn{2}{|c|}{ Invalid } & \multicolumn{2}{|c|}{ Absent } & \multicolumn{2}{|c|}{ Validity Effect } \\
\hline & RT & $\mathrm{PE}$ & RT & PE & $\mathrm{RT}$ & $\mathrm{PE}$ & $\mathrm{RT}$ & PE \\
\hline \multicolumn{9}{|l|}{ Set size 4} \\
\hline Parallel & 532 & $5.1 \%$ & 562 & $7.0 \%$ & 554 & $5.3 \%$ & $30^{*}$ & $1.9 \%$ \\
\hline Serial & 608 & $6.2 \%$ & 642 & $7.6 \%$ & 629 & $6.0 \%$ & $34^{*}$ & $1.4 \%$ \\
\hline \multicolumn{9}{|l|}{ Set size 8} \\
\hline Parallel & 551 & $5.8 \%$ & 590 & $7.0 \%$ & 584 & $6.8 \%$ & $38^{*}$ & $1.3 \%$ \\
\hline Serial & 672 & $8.7 \%$ & 725 & $10.4 \%$ & 712 & $9.3 \%$ & $53^{*}$ & $1.7 \%$ \\
\hline
\end{tabular}

Validity effects were calculated as invalid minus valid. Asterisks indicate cue validity effects significantly greater than zero $(p<.05)$.

The data indicate that our task-irrelevant abrupt-onset cues captured attention. Participants showed cue validity effects, responding more slowly on invalid trials $(630 \mathrm{~ms})$ than on valid trials $(591 \mathrm{~ms}), F(1,57)=66.751, p<.001, \eta^{2}$ $=.539$. These cue validity effects were significantly larger at set size $8(46 \mathrm{~ms})$ than at set size $4(32 \mathrm{~ms}), F(1,57)=$ $6.281, p<.05, \eta^{2}=.099$.

The critical question was whether irrelevant onsets would capture attention only under parallel search. Clearly this was not the case; cue validity effects were not greater under parallel search $(34 \mathrm{~ms})$ than under serial search $(44 \mathrm{~ms}), F(1$, $57)=1.994, p>.10, \eta^{2}=.034$. Note that this nonsignificant trend (larger cue validity effects under the serial than the parallel condition) is actually in the wrong direction, relative to that predicted by the strong version of Theeuwes's (2004) original attentional-window account. Also, the three-way interaction of search condition, set size, and validity was nonsignificant, $F(1,57)=1.03, p>.10, \eta^{2}=.018$. Preplanned $t$ tests revealed that the cue validity effects at each set size and search condition were significant (see Table 3). Cue validity effects were significant under parallel search at set sizes of both four and eight, $t(57)=5.40, p<.001$, and $t(57)=6.32, p$ $<.001$. These effects were also significant under serial search at set sizes of both four and eight, $t(57)=6.49, p<.001$, and $t$ $(57)=5.31, p<.001$. All together, these results suggest that search mode did not affect capture by abrupt onsets. Instead, a nonsignificant trend suggested that capture may actually be greater under serial search.

The same three-way ANOVA was conducted on mean error rates on cue-present trials as well. Participants made more errors in the serial $(8.2 \%)$ than in the parallel $(6.2 \%)$ condition, $F(1,57)=6.77, p<.05, \eta^{2}=.106$. They also made more errors at set size $8(8.0 \%)$ than at set size 4 $(6.5 \%), F(1,57)=10.439, p<.01, \eta^{2}=.156$. The participants also made more errors on invalid trials $(8.0 \%)$ than on valid trials $(6.4 \%), F(1,57)=12.750, p=.001, \eta^{2}=$ .183 , and had steeper error rate slopes (akin to search slope) in the serial condition $(0.7 \%$ per item) than in the parallel condition $\left(0.1 \%\right.$ per item), $F(1,57)=4.78, p<.05, \eta^{2}=$ .077. All other interactions were nonsignificant.

Experiments 1 and 2 replicated previous results, showing greater capture effects by color singletons under parallel search (Belopolsky et al., 2007). However, in this experiment, we found, if anything, the opposite effect for taskirrelevant abrupt onsets: The nonsignificant trend went in the wrong direction, hinting that there might be even greater capture under serial than under parallel search.

\section{Experiment 4}

In Experiment 3, we found no evidence of enhanced capture by onset precues (150-ms cue-to-target stimulus onset asynchrony $[\mathrm{SOA}]$ ) under parallel search. Note, however, that many studies demonstrating capture by abrupt onsets have presented the onset simultaneously with the search array (e.g., Franconeri \& Simons, 2003; Jonides \& Yantis, 1988). Although we see no obvious reason why this should matter, we wanted to replicate our results under the conditions most commonly studied. In this experiment, therefore,

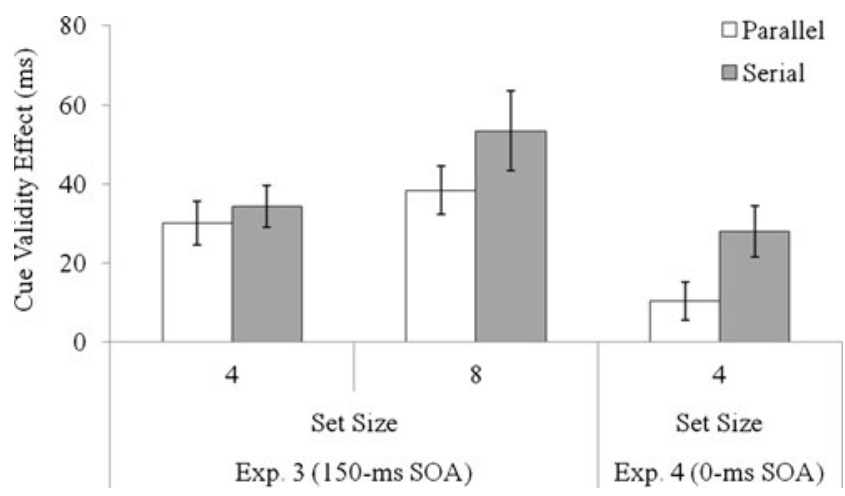

Fig. 4 Cue validity effects for irrelevant abrupt onsets by search condition and set size in Experiments 3 and 4. Cue validity effects were calculated as the invalid minus the valid mean. Bars represent the standard errors of the means 
we assessed whether capture by onset cues appearing simultaneous with the search array ( 0 -ms SOA) would be enhanced under serial search.

\section{Methods}

Participants A new sample of 39 University of New Mexico students participated for course credit. Two of the participants were excluded from the analysis because of unusually high error rates (more than $20 \%$ ).

Apparatus, stimuli, and procedure This experiment was nearly identical to Experiment 3, except that the precue $(100 \mathrm{~ms})$ and the intermediate frame $(50 \mathrm{~ms})$ were removed. Instead, the onset cue appeared simultaneously with the search display. When present, the onset cue appeared at the target location on $25 \%$ of trials (i.e., was nonpredictive). Also, set size was not manipulated; all displays contained only four placeholders, and the search arrays contained only four letters, as in Experiment 2.

\section{Results and discussion}

The data analysis was similar to that of the previous experiments. Application of the RT cutoffs ( $<200$ or $>2,000 \mathrm{~ms})$ eliminated $0.3 \%$ of trials from the RT and error rate analyses. The resulting mean RTs and error rates are shown in Table 4 . The cue validity effects by condition are shown in Fig. 4.

First, we assessed whether our search manipulation was effective on cue-absent trials. A preplanned $t$ test revealed that participants did in fact respond more quickly in the parallel condition $(532 \mathrm{~ms})$ than in the serial condition (586 ms) when the cue was absent, $t(36)=9.27, p<.001$. Second, a two-way within-subjects ANOVA was conducted on mean RTs with the factors Search Condition (parallel vs. serial) and Cue Validity (invalid vs. valid). Participants were again significantly faster in the parallel search condition $(535 \mathrm{~ms})$ than in the serial search condition $(594 \mathrm{~ms}), F(1$, $36)=84.853, p<.001, \eta^{2}=.702$. They responded more slowly following invalid onset cues $(575 \mathrm{~ms})$ than following valid cues $(556 \mathrm{~ms}), F(1,36)=20.11, p<.001, \eta^{2}=.358$, indicating attention capture by onsets.
Again, the main point of this study was to determine whether capture by irrelevant onsets was greater under serial than under parallel search. The participants produced significantly greater cue validity effects under serial search $(28 \mathrm{~ms})$ than under parallel search $(10 \mathrm{~ms}), F(1,36)=5.494, p<.05$, $\eta^{2}=.132$, confirming the trend observed in Experiment 3 . Preplanned $t$ tests revealed that cue validity effects were significant under both the parallel and serial conditions, $t(36)=2.149, p<.05$, and $t(36)=4.321, p<.001$.

The same two-way ANOVA was conducted on mean error rates as well. Participants made significantly more errors under serial search $(10.1 \%)$ than under parallel search $(7.9 \%), F(1,36)=8.493, p=.01, \eta^{2}=.279$. All other main effects and interactions were nonsignificant.

To summarize, we investigated whether Experiment 3 (which a showed a trend toward greater capture under serial search) would be replicated, even when the irrelevant abrupt onset appeared with the search array (0-ms SOA). We once again found stronger cue validity effects by abrupt onsets under serial than under parallel search, and this time the trend was statistically significant. In fact, cue validity effects by abrupt onsets were minuscule in the parallel condition (only $10 \mathrm{~ms}$ ). This finding directly contradicts Theeuwes's attentional-window account of capture.

\section{Experiment 5}

Experiments 1-4 showed that search mode can significantly influence capture by abrupt onsets and color singletons. We did find the typical steeper search slopes for serial than for parallel search in Experiment 3. However, one could argue that the cue appeared on only a portion of trials in that experiment, possibly adding noise to the search slopes. In this control experiment, we removed the precue so as to provide a purer assessment of whether our search mode manipulation was effective.

Method

Participants A new sample of 25 University of New Mexico students participated for course credit. Two of the participants

Table 4 Mean response times (RTs, in milliseconds) and percentages of errors (PEs) as a function of search mode (parallel vs. serial) and cue validity (valid vs. invalid) for Experiment 4

\begin{tabular}{|c|c|c|c|c|c|c|c|c|}
\hline \multirow[t]{2}{*}{ Trial Type } & \multicolumn{2}{|c|}{ Valid } & \multicolumn{2}{|c|}{ Invalid } & \multicolumn{2}{|c|}{ Absent } & \multicolumn{2}{|c|}{ Validity Effect } \\
\hline & $\mathrm{RT}$ & PE & $\mathrm{RT}$ & $\mathrm{PE}$ & $\mathrm{RT}$ & $\mathrm{PE}$ & $\mathrm{RT}$ & $\mathrm{PE}$ \\
\hline Serial & 576 & $9.7 \%$ & 604 & $10.1 \%$ & 586 & $10.4 \%$ & $28^{*}$ & $0.4 \%$ \\
\hline Parallel & 529 & $8.5 \%$ & 539 & $7.9 \%$ & 532 & $7.6 \%$ & $10^{*}$ & $0.6 \%$ \\
\hline
\end{tabular}

Validity effects were calculated as invalid minus valid. Asterisks indicate cue validity effects significantly greater than zero $(p<.05)$. 
were excluded from the analysis because of an unusually high error rate (more than $20 \%$ ). All of the participants had normal color vision, as assessed by the Ishihara color vision test. They also self-reported normal or corrected-to-normal visual acuity.

Apparatus, stimuli, and procedure This experiment was nearly identical to Experiment 3, except that the precue $(100 \mathrm{~ms})$ and the intermediate frame $(50 \mathrm{~ms})$ were removed. Set size was manipulated by trials. Again, search display type (parallel vs. serial) was varied within participants. The experiment was divided into two session halves, one for each search condition, and their order was counterbalanced across participants. During each session half, participants performed a practice block of 36 trials, followed by five blocks of 72 trials (360 total).

\section{Results and discussion}

The data analysis was similar to that of Experiment 4. Application of the RT cutoffs ( $<200$ or $>2,000 \mathrm{~ms})$ eliminated $0.3 \%$ of trials from the RT and error rate analyses. The resulting mean RTs and error rates are shown in Table 5.

We conducted a two-way ANOVA on mean RTs with two factors: Search Condition (parallel vs. serial) and Set Size (4 vs. 8). Participants generally responded faster in the parallel $(524 \mathrm{~ms})$ than in the serial $(599 \mathrm{~ms})$ condition, $F(1,22)=$ $56.512, p<.001, \eta^{2}=.72$. They were also faster at set size 4 (534 ms) than at set size $8(590 \mathrm{~ms}), F(1,22)=333.603, p<$ $.001, \eta^{2}=.938$. Most importantly, search slopes were more than twice as steep in the serial condition (19.2 ms per item) than in the parallel condition $(8.7 \mathrm{~ms}$ per item), $F(1,22)=$ $65.958, p<.001, \eta^{2}=.75$. This set size by search condition interaction suggests that our manipulation of search mode was in fact successful.

The same ANOVA was applied to mean error rates. Participants generally made slightly more errors at set size $8(9.8 \%)$ than at set size $4(8.5 \%), F(1,22)=6.25, p<.05$, $\eta^{2}=.229$. All other main effects and interactions were nonsignificant.

Table 5 Mean response times (RTs, in milliseconds) and percentages of errors (PEs) as a function of search mode (parallel vs. serial) and set size (4 vs. 8) for Experiment 5

\begin{tabular}{lllllll}
\hline Trial Type & 4 & & & 8 & & \multirow{2}{*}{ Search Slope } \\
\cline { 2 - 3 } & RT & PE & & RT & PE & \\
\hline Parallel & 507 & $8.1 \%$ & & 542 & $9.0 \%$ & 8.6 \\
Serial & 561 & $9.0 \%$ & & 638 & $10.6 \%$ & 19.2 \\
\hline
\end{tabular}

Search slopes were calculated as set size 8 minus set size 4 and then divided by four.

\section{General discussion}

Researchers currently debate whether purely stimulusdriven attentional capture is possible. While one line of research has provided evidence that attentional capture is strictly goal-driven (Atchley, Kramer, \& Hillstrom, 2000; Folk \& Remington, 1998; Folk et al., 1992; Folk, Remington, \& Wright, 1994; Gibson \& Kelsey, 1998; Lien, Ruthruff, \& Cornett, 2010; Lien, Ruthruff, Goodin, \& Remington, 2008; Lien, Ruthruff, \& Johnston, 2010), another line has routinely provided evidence of stimulusdriven capture (Belopolsky \& Theeuwes, 2010; Belopolsky et al., 2007; Franconeri \& Simons, 2003; Theeuwes, 1992, 2004, 2010; Yantis \& Jonides, 1984). To reconcile these conflicting results, Theeuwes $(1991,2004,2010)$ proposed that stimulus-driven capture is possible only under parallel search, when participants employ a diffuse attentional window. Perhaps serial search is too slow or too deliberate to be strongly influenced by task-irrelevant salience; for example, the "pull" from salient items might wear off over time, or be ignored when participants choose a scan path in advance. In fact, a few studies have supported this claim with color singletons (Belopolsky \& Theeuwes, 2010; Belopolsky et al., 2007; Theeuwes, 1992, 1994). However, the effect of search mode has thus far been demonstrated using a single paradigm (the additional-singleton paradigm) with some notable drawbacks, and using a narrow range of salient stimuli (always color singletons). Our aim was to determine whether the effect of search mode generalizes to other salient stimuli and paradigms, particularly those that allow for a more definitive assessment of whether spatial attention was captured.

In the present experiments, we used a precuing paradigm and manipulated search mode via color space. In Experiment 1, with set size 8, we found that capture by taskirrelevant color singletons was indeed greater under parallel search (cue validity effect of $27 \mathrm{~ms}$ ) than serial search $(9 \mathrm{~ms})$. In Experiment 2, we replicated these effects at the smaller set size of 4 that is typical of the precuing paradigm (e.g., Folk et al., 1992). So, capture by irrelevant color singletons does seem to depend on a parallel search mode.

When we investigated capture by abrupt onsets, however, parallel search mode was not necessary for capture. In Experiment 3, we found no evidence that capture by abrupt onsets was greater under parallel search (cue validity effect $34 \mathrm{~ms}$ ) than under serial search (44 ms). In fact, marginally significant trends in the cue validity effects suggested that capture was promoted under serial search. In Experiment 4, we assessed attentional capture with abrupt-onset cues appearing simultaneously with, rather than before (as with a precue), the search display. Here, we found substantially larger capture effects by abrupt onsets under serial search $(28 \mathrm{~ms})$ than under parallel search $(10 \mathrm{~ms})$. 
All together, these findings argue against the strong version of the attentional-window account, which proposes that a diffuse attentional window (i.e., parallel search) inherently promotes capture by all salient stimuli (e.g., Theeuwes, 1991). Instead, the pattern of results is consistent with a weaker version of the attentional-window account, which asserts that abrupt onsets are somehow special (e.g., Belopolsky et al., 2007; Theeuwes, 2010).

\section{Relation to previous research}

Our finding that capture effects are quite different for color singletons and abrupt onsets has an unanticipated implication for previous studies comparing onsets and color singletons (Franconeri, Hollingworth, \& Simons, 2005; Franconeri \& Simons, 2003; Jonides \& Yantis, 1988). These studies have routinely demonstrated that abrupt onsets (and other dynamic stimuli) are able to capture attention more strongly than color singletons. The catch is that these studies typically use designs that encourage serial search (e.g., Franconeri \& Simons, 2003). Our findings suggest that, had the authors instead used displays that encouraged parallel search, the difference in capture might have disappeared.

Our finding of greater capture by irrelevant color singletons under parallel search might be criticized as resulting from singleton detection mode. Bacon and Egeth (1994) argued that participants can use two distinct search modes: a singleton detection mode, where participants search broadly for singletons, and a feature search mode, where participants search for a specific feature (for a recent review, see Egeth, Leonard, \& Leber, 2010). Capture, they claimed, occurs only under singleton detection mode, when participants have an attentional set for any feature singleton. In the parallel search condition, a singleton detection theorist might claim that we encouraged our participants to use a singleton detection mode and to search more generally for color-space singletons.

However, it is unlikely that participants were using singleton detection mode. We took precautions to discourage this mode by including an additional color-space singleton distractor (a white letter) and two different distractor colors in all displays. Also, participants always showed greater capture for relevant than for irrelevant cues, even under parallel search. Singleton detection accounts would seem to predict no differences in capture by either cue type, because both match the presumed goal of finding a feature singleton. Moreover, the results of Experiments 3 and 4 (with the same task as in Exps. 1 and 2) are entirely inconsistent with singleton detection mode accounts. Such accounts would naturally predict greater capture for abrupt onsets under parallel search (supposedly encouraging singleton detection mode) than under serial search (supposedly encouraging feature search), yet we observed the opposite data pattern.

\section{Relevance versus salience}

The present data provide evidence that irrelevant salient stimuli can capture attention to some degree, even when they do not resemble the target. But what is more important for attentional capture, relevance or salience? Many experiments demonstrating capture have not included relevant cues. So, Experiments 1 and 2 give us a unique opportunity to compare capture by relevant and irrelevant cues. In these experiments, it was clear that relevant cues captured attention much more strongly. Relevant color cues captured attention regardless of search mode, unlike irrelevant cues. Even under parallel search, the pooled cue validity effects (Table 6) show that the irrelevant-cue validity effects $(25.7 \mathrm{~ms})$ were only about $47 \%$ the size of those produced by relevant orange cues $(54.9 \mathrm{~ms})$. This finding casts doubt on attentional-window accounts claiming that top-down selectivity disappears under parallel search (Belopolsky et al., 2007). The present data suggest that, at most, top-down selectivity is reduced under parallel search.

Unlike the present study, many previous precuing studies have reported no evidence of capture from irrelevant color singletons (Folk \& Remington, 1998; Folk et al., 1992; Folk et al., 1994; Gibson \& Kelsey, 1998; Lien, Ruthruff, \& Cornett, 2010; Lien et al., 2008; Lien, Ruthruff, \& Johnston, 2010). To further investigate this discrepancy, we performed a finer-grained two-way ANOVA with the factors Search Condition (parallel vs. serial) and Irrelevant-Cue Color (blue/green vs. yellow/red) on the cue validity effects pooled across Experiments 1 and 2. Cue validity effects were greater for the parallel conditions $(25.7 \mathrm{~ms})$ than for the serial conditions $(8.1 \mathrm{~ms}), F(1,97)=11.22, p=.001, \eta^{2}=$ .104 , and greater for yellow/red cues $(26.9 \mathrm{~ms})$ than for blue/ green cues $(6.9 \mathrm{~ms}), F(1,97)=16.82, p<.001, \eta^{2}=.148$. The interaction between these variables was nonsignificant, $F(1$, $97)=0.347, p>.10, \eta^{2}=.004$. So, capture by irrelevant cues depended strongly on both search mode and the similarity of the irrelevant cue color to the target.

One explanation for this pattern of results is that participants slightly broadened their attentional set under parallel search to include irrelevant colors similar to the target color; because the distractors in the target display were never close

Table 6 Mean cue validity effects (invalid minus valid; in milliseconds) as a function of search mode (parallel vs. serial) and cue type (relevant vs. irrelevant) for the pooled data of Experiments 1 and 2

\begin{tabular}{|c|c|c|c|c|}
\hline & \multirow{2}{*}{$\begin{array}{l}\text { Relevant } \\
\text { Orange }\end{array}$} & \multicolumn{3}{|l|}{ Irrelevant } \\
\hline & & Yellow \& Red & Blue \& Green & All \\
\hline Parallel & 54.9 & 37.2 & 14.3 & 25.7 \\
\hline Serial & 66.6 & 16.7 & -0.5 & 8.1 \\
\hline
\end{tabular}


to the target color in that condition, they could afford to do so. On this view, capture by irrelevant color cues would still be goal-driven. But, because there is no independent measure of attentional set in the precuing paradigm, it is difficult to determine exactly what participants were looking for. Note that such a goal-driven account would have difficulty explaining the small but significant cue validity effects by blue and green cues under parallel search (14.3 ms).

Additional factors may have also increased the probability of capture by salient irrelevant stimuli (color singletons and abrupt onsets) in the present study. For example, large set sizes (as in Exps. 1 and 3) may enhance the costs and benefits of capture, without necessarily increasing the probability of capture (Yeh \& Liao, 2008). Also, abrupt-onset cues appeared only on $50 \%$ of the trials, and there is some evidence that salient stimuli capture attention more effectively when presented rarely (Neo \& Chua, 2006), perhaps because there is less incentive to inhibit them.

Parallel versus serial search revisited

Many researchers have pointed out that it is difficult to unambiguously determine whether search is parallel or serial (Moore \& Wolfe, 2001; Palmer, 1995; Pashler, 1987; Townsend, 1971, 1976, 1990; Wolfe, 1994, 1998a, 1998b). Although steep and flat search slopes are certainly consistent with the "serial" and "parallel" distinctions, respectively, alternative explanations are logically tenable. For example, the typical linearly increasing search slopes that are indicative of serial search could be a result of a limited-capacity parallel search (Mordkoff \& Yantis, 1993; Townsend, 1971, 1976, 1990) or decision noise (Palmer, 1995; Palmer, Verghese, \& Pavel, 2000). Indeed, actual data do not necessarily reveal a dichotomous distinction between serial- and parallel-search slopes (Wolfe, 1998b).

Regardless of these criticisms, the present experiments were designed to test Theeuwes's attentional-window account, which presumes the existence of two different search modes. Even if one assumes that no distinct search modes exist, the present data still show that making search more difficult (i.e., "more serial" or "less efficient") can strongly influence attentional capture, and therefore deserves more study.

\section{Concluding remarks}

Previous researchers have argued that capture occurs only under parallel search with a diffuse attentional window (Theeuwes, 2004). For example, a bright billboard might capture attention only when we are searching a scene in parallel for potential hazards, but not when serially searching signs for a particular street name. Previous studies have supported this proposition in the case of color singletons, often using the additional-singleton paradigm (e.g., Theeuwes,
1992, 1994). However, these studies did not examine other types of salient stimuli, such as abrupt onsets, or use alternative paradigms that can more reliably measure shifts of spatial attention.

We investigated this issue using a precuing paradigm, with easy and difficult searches (intended to encourage parallel and serial search, respectively). For color singletons, we demonstrated greater effects of capture under parallel than under serial search. However, unlike previous studies showing this effect, the capture effects here were confirmed using a reliable indicator of the capture of spatial attentioncue validity effects. Nevertheless, we found the opposite pattern of results when we examined capture by abrupt onsets: Capture effects were actually greater for serial than for parallel search. These results do not support strong versions of the attentional-window theory, which claim that capture by any salient stimulus requires parallel search. Instead, abrupt onsets and color singletons seem to be oppositely affected by search mode. These results are roughly consistent with weak versions of the attentional-window theory, which adds the provision that abrupt onsets are an exception and can capture attention even under serial search. The present findings also argue against the strong claim that, under parallel search, attentional capture is driven only by bottom-up salience. We found that relevant cues produced much greater capture effects than did irrelevant cues, even under parallel search.

\section{References}

Atchley, P., Kramer, A. F., \& Hillstrom, A. P. (2000). Contingent capture for onsets and offsets: Attentional set for perceptual transients. Journal of Experimental Psychology: Human Perception and Performance, 26, 594-606.

Bacon, W. F., \& Egeth, H. E. (1994). Overriding stimulus-driven attentional capture. Perception \& Psychophysics, 55, 485-496. doi:10.3758/BF03205306

Becker, S. I. (2007). Irrelevant singletons in pop-out search: Attentional capture or filtering costs? Journal of Experimental Psychology: Human Perception and Performance, 33, 764-787.

Belopolsky, A. V., \& Theeuwes, J. (2010). No capture outside the attentional window. Vision Research, 50, 2543-2550. doi:10.1016/ j.visres.2010.08.023

Belopolsky, A. V., Zwaan, L., Theeuwes, J., \& Kramer, A. F. (2007). The size of an attentional window modulates attentional capture by color singletons. Psychonomic Bulletin \& Review, 14, 934 938. doi:10.3758/BF03194124

Egeth, H. E., Leonard, C. J., \& Leber, A. B. (2010). Why salience is not enough: Reflections on top-down selection in vision. Acta Psychologica, 135, 130-132.

Folk, C. L., \& Remington, R. (1998). Selectivity in distraction by irrelevant featural singletons: Evidence for two forms of attentional capture. Journal of Experimental Psychology: Human Perception and Performance, 24, 847-858. doi:10.1037/00961523.24.3.847

Folk, C. L., \& Remington, R. W. (2010). A critical evaluation of the disengagement hypothesis. Acta Psychologica, 135, 103-105. 
Folk, C. L., Remington, R. W., \& Johnston, J. C. (1992). Involuntary covert orienting is contingent on attentional control settings. Journal of Experimental Psychology: Human Perception and Performance, 18, 1030-1044. doi:10.1037/00961523.18.4.1030

Folk, C. L., Remington, R. W., \& Wright, J. H. (1994). The structure of attentional control: Contingent attentional capture by apparent motion, abrupt onset, and color. Journal of Experimental Psychology: Human Perception and Performance, 20, 317-329. doi:10.1037/0096-1523.20.2.317

Franconeri, S. L., Hollingworth, A., \& Simons, D. J. (2005). Do new objects capture attention? Psychological Science, 16, 275-281. doi:10.1111/j.0956-7976.2005.01528.x

Franconeri, S. L., \& Simons, D. J. (2003). Moving and looming stimuli capture attention. Perception \& Psychophysics, 65, 999-1010. doi:10.3758/BF03194829

Gibson, B. S., \& Kelsey, E. M. (1998). Stimulus-driven attentional capture is contingent on attentional set for displaywide visual features. Journal of Experimental Psychology: Human Perception and Performance, 24, 699-706.

Jonides, J., \& Yantis, S. (1988). Uniqueness of abrupt visual onset in capturing attention. Perception \& Psychophysics, 43, 346-354. doi:10.3758/BF03208805

Lamy, D., \& Egeth, H. E. (2003). Attentional capture in singletondetection and feature-search modes. Journal of Experimental Psychology: Human Perception and Performance, 29, 1003-1020.

Lien, M.-C., Ruthruff, E., \& Cornett, L. (2010a). Attentional capture by singletons is contingent on top-down control settings: Evidence from electrophysiological measures. Visual Cognition, 18, 682-727.

Lien, M.-C., Ruthruff, E., Goodin, Z., \& Remington, R. W. (2008). Contingent attentional capture by top-down control settings: Converging evidence from event-related potentials. Journal of Experimental Psychology: Human Perception and Performance, 34, 509-530.

Lien, M.-C., Ruthruff, E., \& Johnston, J. C. (2010b). Attentional capture with rapidly changing attentional control settings. Journal of Experimental Psychology: Human Perception and Performance, 36, 1-16. doi:10.1037/a0015875

Moore, C. M., \& Wolfe, J. M. (2001). Getting beyond the serial/ parallel debate in visual research: A hybrid approach. In K. Shapiro (Ed.), The limits of attention: Temporal constraints in human information processing (pp. 178-198). New York: Oxford University Press.

Mordkoff, J. T., \& Yantis, S. (1993). Dividing attention between color and shape: Evidence of coactivation. Perception \& Psychophysics, 53, 357-366.

Neo, G., \& Chua, F. K. (2006). Capturing focused attention. Perception \& Psychophysics, 68, 1286-1296. doi:10.3758/ BF03193728

Palmer, J. (1995). Attention in visual search: Distinguishing four causes of a set-size effect. Current Directions in Psychological Science, 4, 118-123. doi:10.1111/1467-8721.ep10772534

Palmer, J., Verghese, P., \& Pavel, M. (2000). The psychophysics of visual search. Vision Research, 40, 1227-1268.
Pashler, H. (1987). Detecting conjunctions of color and form: Reassessing the serial search hypothesis. Perception \& Psychophysics, $41,191-201$.

Rauschenberger, R. (2003). Attentional capture by auto- and allo-cues. Psychonomic Bulletin \& Review, 10, 814-842. doi:10.3758/ BF03196545

Schreij, D., Owens, C., \& Theeuwes, J. (2008). Abrupt onsets capture attention independent of top-down control settings. Perception \& Psychophysics, 70, 208-218. doi:10.3758/PP.70.2.208

Schreij, D., Theeuwes, J., \& Olivers, C. N. L. (2010). Irrelevant onsets cause inhibition of return regardless of attentional set. Attention, Perception, \& Psychophysics, 72, 1725-1729.

Simons, D. J., \& Chabris, C. F. (1999). Gorillas in our midst: Sustained inattentional blindness for dynamic events. Perception, 28, 1059 1074. doi: $10.1068 / \mathrm{p} 2952$

Theeuwes, J. (1991). Exogenous and endogenous control of attention: The effect of visual onsets and offsets. Perception \& Psychophysics, 49, 83-90. doi:10.3758/BF03211619

Theeuwes, J. (1992). Perceptual selectivity for color and form. Perception \& Psychophysics, 51, 599-606. doi:10.3758/BF03211656

Theeuwes, J. (1994). Stimulus-driven capture and attentional set: Selective search for color and visual abrupt onsets. Journal of Experimental Psychology: Human Perception and Performance, 20, 799-806. doi:10.1037/0096-1523.20.4.799

Theeuwes, J. (2004). Top-down search strategies cannot override attentional capture. Psychonomic Bulletin \& Review, 11, 65-70. doi:10.3758/BF03206462

Theeuwes, J. (2010). Top-down and bottom-up control of visual selection. Acta Psychologica, 135, 77-99. doi:10.1016/j.actpsy.2010.02.006

Townsend, J. T. (1971). A note on the identifiability of parallel and serial processes. Perception \& Psychophysics, 10, 161-163.

Townsend, J. T. (1976). Serial and within-stage independent parallel model equivalence on the minimum completion time. Journal of Mathematical Psychology, 14, 219-238.

Townsend, J. T. (1990). Serial vs. parallel processing: Sometimes they look like Tweedledum and Tweedledee but they can (and should) be distinguished. Psychological Science, 1, 46-54. doi:10.1111/ j.1467-9280.1990.tb00067.x

Treisman, A. M., \& Gelade, G. (1980). A feature-integration theory of attention. Cognitive Psychology, 12, 97-136. doi:10.1016/00100285(80)90005-5

Wolfe, J. M. (1994). Guided Search 2.0: A revised model of visual search. Psychonomic Bulletin \& Review, 1, 202-238. doi:10.3758/ BF03200774

Wolfe, J. M. (1998a). Visual search. In H. Pashler (Ed.), Attention (pp. 13-73). Hove: Psychology Press.

Wolfe, J. M. (1998b). What can 1 million trials tell us about visual search? Psychological Science, 9, 33-39. doi:10.1111/1467-9280.00006

Yantis, S., \& Jonides, J. (1984). Abrupt visual onsets and selective attention: Evidence from visual search. Journal of Experimental Psychology: Human Perception and Performance, 10, 601-621. doi:10.1037/0096-1523.10.5.601

Yeh, S.-L., \& Liao, H.-I. (2008). On the generality of the contingent orienting hypothesis. Acta Psychologica, 129, 157-165. doi:10.1016/j.actpsy.2008.05.008 